\title{
Les sciences, les philosophies et la pensée : une affaire de justice
}

Un vieux thème flotte à nouveau dans l'air du temps, revues et ouvrages de vulgarisation présentent la science comme devant être accompagnée de pensée ou de civilisation : «Science avec conscience » (Edgar Morin), «Et si les sciences devenaient un jour civilisées?» (Isabelle Stengers), «Savant en manque de philosophie » (Dominique Lecourt). Ces expressions supposent les sciences ignorantes des choses humaines et devant être complétées par des considérations plus nobles, de nature philosophique. Cette idée, qui semble aller de soi, est nuisible à la science, à la philosophie, à la pensée. Nous voulons donc la reconsidérer, montrer en quoi elle n'est qu'une évidence convenue qu'il faut briser pour avoir une conception plus juste des sciences et de leurs relations aux philosophies. L'évidence porte sur l'idée qu'il faut deux choses distinctes pour donner lieu à une science civilisée ou à une science pensante, comme si celle-ci manquait d'autonomie. Elle repose sur une dissymétrie supposée dans les relations entre sciences et philosophies. Les sciences ont les connaissances et manqueraient de conscience que devrait leur fournir la philosophie, et la philosophie serait dénuée de vraies connaissances, étant supposée générale.

Cette évidence n'est au fond qu'une vieille rengaine. Quelles qu'aient été les modifications et des sciences et des philosophies, elle est restée identique à elle-même ( "Science sans conscience n'est que ruine de l'âme »Rabelais). Cette seule considération devrait suffire à nous alerter. Admettons que toute activité, quelle qu'elle soit, doive être accompagnée de conscience et doive renoncer à son autonomie pour devenir «humaine». Mais alors, pourquoi réserver ce jugement aux seules sciences? Pourquoi ne pas demander si la philosophie est elle-même civilisée, si l'éthique n'est pas en manque de conscience, et la philosophie pratiquée avec éthique? On avait naguère trouvé tout à fait naturel de parler de la "philosophie spontanée des savants » pour caractériser leurs généralisations peu contrôlées à partir des connaissances (Louis Althusser); mais pourquoi ne pas prêter attention à la "philosophie spontanée des philosophes », qui existe aussi, qui fait peut-être même l'essentiel de toute pratique philosophique? Il y a là une inégalité dans la distribution de la pensée. En réalité, cette dissymétrie repose sur une différence des sciences et des philosophies dans leur rapport au réel. La pratique scientifique admet qu'il y a du réel, aucun scientifique ne pourrait travailler s'il ne supposait pas un objet d'étude, c'est évident, mais aussi, plus profondément, s'il ne convenait pas que cet objet a quelque relation, même indirecte, à un réel. Mais le scientifique ne fait pas d'hypothèse sur les relations entre son objet et le réel, il les admet et les met en œuvre. Le philosophe, au contraire, tente d'expliciter et de rationaliser son rapport au réel qui est en quelque sorte son «objet », et c'est dans cette explicitation qu'il construit, selon les cas, le «vrai » ou la «vérité ». Mais, du coup, par cette explicitation, il juge que ce réel coappartient à la philosophie et que celle-ci peut le transformer.

Cette dissymétrie doit être corrigée. Il faut rétablir la justice entre les disciplines. Ce n'est pas parce que la science peut s'occuper d'objets ordinaires qu'elle est ordinaire et sans conscience, et parce que la philosophie a pour objet la généralité qu'elle est elle-même générale et sans connaissances. Elle est une technique des généralités, et non pas une généralité sur les sciences. La pratique actuelle de la modélisation rend urgente cette réparation, en sciences tout d'abord, parce que la construction de modèles conduit à articuler des fragments de disciplines différentes en fonction d'un même problème, mais aussi en philosophie. Comment, en effet, admettre la multiplicité des philosophies, qui semble actuellement aller de soi, si l'on croit toujours que chacune d'entre elles a un rapport privilégié au réel? Tout cela est à repenser, les relations entre sciences et philosophies doivent devenir plus démocratiques, les premières n'ont pas à refuser aux 
philosophies les connaissances, et les secondes n'ont pas à survoler les premières en leur dictant le «bon choix » du réel.

Il ne suffit pas, pour aller vers plus de symétrie, d'affirmer que «la science pense ». Il faut modifier notre représentation de la pensée, ne pas en faire un après-coup des connaissances, quelque chose comme leur conscience. La pensée, au contraire, précède les disciplines, elle est vide de leur contenu, elle est une force qui contribue à leur invention. Il n'y a pas de disciplines favorisées du point de vue de la pensée, si ce n'est, peut-être, justement celles qui ont su simplement admettre un rapport au réel. La question de "civilisation » consiste à repenser les relations entre disciplines, sciences, arts, technologies, philosophies, éthiques, en fonction de cette donnée. Le savoir de ces relations est, par définition, un nouvel « objet complexe » qui ne peut se réduire à l'une ou l'autre des disciplines.
Pourquoi a-t-on accusé les sciences plutôt que les autres modes de la pensée de manquer de civilisation ou de conscience? L'explication par les suites technologiques est trop évidente et très insuffisante. Il est aujourd'hui essentiel de comprendre que le simple rapport au réel est une condition de la pensée qui n'était pas pensable dans la philosophie classique qui n'explicitait pas ses gestes d'autoconstitution. Que les philosophies contemporaines soient susceptibles de laisser être ce rapport sans chercher à le modifier est essentiel, et demande un véritable effort d'invention. Une juste posture oblige à transformer de fond en comble nos idées des sciences et des philosophies dans leur rapport à la pensée.

Anne-Françoise Schmid

To access this journal online: www.edpsciences.org 